\title{
Induction of $\beta$-lactamase production in Aeromonas hydrophila is responsive to $\beta$-lactam-mediated changes in peptidoglycan composition
}

Correspondence

Matthew B. Avison

Matthewb.Avison@bristol.ac.uk

Received 2 October 2009

Revised 15 March 2010

Accepted 23 April 2010
Amy E. Tayler, ${ }^{1}$ Juan A. Ayala, ${ }^{2}$ Pannika Niumsup, ${ }^{1} \dagger$ Katrin Westphal, ${ }^{1,3}$ Jenny A. Baker, ${ }^{1}$ Lufei Zhang, ${ }^{1}$ Timothy R. Walsh, ${ }^{1} \ddagger$ Bernd Wiedemann, ${ }^{3}$ Peter M. Bennett ${ }^{1}$ and Matthew B. Avison ${ }^{1}$

\author{
${ }^{1}$ Department of Cellular and Molecular Medicine, University of Bristol, Bristol BS8 1TD, UK \\ ${ }^{2}$ Centro de Biología Molecular 'Severo Ochoa', Consejo Superior de Investigaciones Cientificas, \\ Universidad Autonoma de Madrid, Campus de Cantoblanco, 28049 Madrid, Spain \\ ${ }^{3}$ Department of Pharmaceutical Microbiology, University of Bonn, 53115 Bonn, Germany
}

\section{INTRODUCTION}

$\beta$-Lactam resistance in bacteria is most commonly due to the production of $\beta$-lactamase enzymes. In many organisms, $\beta$-lactamase genes are intrinsic and their expression is induced upon $\beta$-lactam challenge. This is true for several members of the Enterobacteriaceae (Hanson \& Sanders, 1999) and other Gram-negative pathogens such as Pseudomonas aeruginosa (Lodge et al., 1993), Burkholderia cepacia (Trepanier et al., 1997), Stenotrophomonas maltophilia (Okazaki \& Avison, 2008) and Aeromonas spp. (Walsh et al., 1997).

The paradigm mechanism for regulation of $\beta$-lactamase production in Gram-negative bacteria is the AmpR system found in many Enterobacteriaceae, P. aeruginosa, B. cepacia and S. maltophilia (Hanson \& Sanders, 1999; Lodge et al., 1993; Okazaki \& Avison, 2008; Trepanier et al., 1997), which has been most extensively studied in Citrobacter freundii (Hanson \& Sanders, 1999). AmpR is a LysR-type transcriptional regulator, and in the absence of any binding

†Present address: Department of Microbiology and Parasitology, Faculty of Medical Science, Naresuan University, Phitsanuloke 65000, Thailand.

$\ddagger$ Present address: Department of Medical Microbiology, School of Medicine, Cardiff University, Cardiff, UK.

Abbreviations: PBP, penicillin-binding protein; TCS, two-component system. ligand, AmpR is an activator of $\beta$-lactamase gene transcription. However, during normal growth, the AmpR repressor ligand, UDP-muramyl-pentapeptide, is produced as part of the peptidoglycan synthetic pathway. This repressor ligand binds to AmpR, altering its conformation and making it bind DNA in such a way that $\beta$-lactamase gene transcription is repressed. Induction of $\beta$ lactamase production occurs because of the increased concentrations of AmpR activatory ligands - anhydromuramyl-tri-, tetra- or pentapeptides - relative to the concentration of UDP-muramyl-pentapeptide repressor ligand in the cytoplasm. During normal cell growth, the precursors of these activatory ligands are constantly being liberated from peptidoglycan via the actions of the lytic transglycosylases. These precursors then enter the cytoplasm via the permease AmpG (Cheng \& Park, 2002) and are recycled so that their constituents can be used to make new peptidoglycan. This recycling process initially involves degradation of the precursors by NagZ, which releases the activatory ligands (Vötsch \& Templin, 2000), followed by breakdown of the activatory ligands by AmpD (Jacobs et al., 1995). This last process keeps activatory ligand concentration low. However, in the presence of $\beta$-lactam challenge (where synthesis of peptidoglycan stops and its breakdown increases dramatically), activatory ligand concentrations rise and repressor ligand concentration falls. The activatory ligands compete with the repressor ligand 
for AmpR binding, and the result is a return of $A m p R$ to its native state as an activator of $\beta$-lactamase production (Jacobs et al., 1997). The key to finding these AmpR activatory ligands was the discovery of mutants that hyperproduce $\beta$-lactamase due to activatory ligand overabundance, which could then be identified using muropeptide profiling techniques. Such mutants generally have mutations in $a m p D$, which destroy or reduce the activity of its product (Tuomanen et al., 1991). In these mutants, the most abundant activatory ligand is the anhydro-muramyltripeptide; but during $\beta$-lactam challenge, the activatory ligand whose concentration most closely correlates with degree of $\beta$-lactamase induction is the anhydro-muramylpentapeptide (Dietz et al., 1997).

The AmpR paradigm of $\beta$-lactamase regulation does not hold for members of the genus Aeromonas, which produce multiple inducible $\beta$-lactamases (Walsh et al., 1995, 1997). In most species there are three distinct enzymes: a class $B$ strict carbapenemase (CphA, also know as Imi), a class $\mathrm{C}$ strict cephalosporinase (Cep) and a class D strict penicillinase with oxacillin- and cloxacillin-hydrolysing ability (Amp) (Avison et al., 2000a; Niumsup et al., 2003). It has long been known that these enzymes share a common control mechanism since mutants selected for overproduction of Cep (e.g. by using greater than MIC concentrations of cefotaxime) always overproduce Amp and Imi as well (Walsh et al., 1995). The first clue to the identity of the regulatory mechanism used came from an experiment where genomic clones from Aeromonas jandaei capable of conferring a trans-dominant phenotype of $\beta$ lactamase overproduction on a wild-type recipient were identified. These recombinants encoded a putative twocomponent system (TCS) response regulator, which the authors named BlrA (Alksne \& Rasmussen, 1997). This finding led to the hypothesis that $\beta$-lactamase gene expression in Aeromonas spp. is controlled by a TCS. Subsequently, cloning the ampH $\beta$-lactamase gene from Aeromonas hydrophila revealed that blrA is encoded upstream, alongside a gene encoding a putative TCS sensor kinase protein, which has been named BlrB. One $\beta$ lactamase-hyperproducing mutant of $A$. hydrophila strain T429125 has been shown to carry a trans-dominant, activatory mutation within the kinase domain of BlrB, and considerable evidence has now been collected that $\mathrm{BlrAB}$ form a TCS regulator of Amp, Cep and Imi $\beta$ lactamase production in Aeromonas spp. (Niumsup et al., 2003).

Most Gram-negative sensor kinases respond to a change in the concentration of a periplasmic or cytoplasmic ligand, either directly or through some additional protein component (West \& Stock, 2001). Proteins known to interact with $\beta$-lactams have a particular signature of amino acid residues that form a $\beta$-lactam-binding pocket (Sauvage et al., 2008). Detailed analysis of A. hydrophila BlrB failed to locate any suggestion of these conserved residues, so it has been concluded that BlrB does not sense $\beta$-lactam challenge directly in Aeromonas spp. This situation is reminiscent of the AmpR paradigm, above, so we have hypothesized that the BlrB activatory ligand is a molecule whose concentration increases upon $\beta$-lactam challenge, and possibly a peptidoglycan constituent (Niumsup et al., 2003). Our current model for $\beta$-lactamase induction in Aeromonas spp. is summarized in Fig. 1. In the work reported in this paper, we set out to develop this model by learning more about what the BlrB activatory ligand is.

\section{METHODS}

Bacterial strains. We chose to work with the well-characterized Aeromonas sp. strain 163a. This has previously been identified as Aeromonas sobria using biochemical testing (Walsh et al., 1995). However, during the course of this work we were alerted to the fact that this may not be the correct speciation for this strain. To definitively identify the species, we used 16S rRNA and rpoB sequencing according to the methods described previously (Küpfer et al., 2006). This showed that $163 \mathrm{a}$ is actually an A. hydrophila strain, its $16 \mathrm{~S}$ rRNA and $r p o B$ sequences being $100 \%$ identical to the $A$. hydrophila ATCC 7966 type strain sequence (Seshadri et al., 2006).

Transposon-insertion mutagenesis to generate $\beta$-lactamasehyperproducing mutants. Plasmid pUT-MiniKm-2 (de Lorenzo et al., 1990) was maintained in Escherichia coli strain S17- $\lambda$ pir (Simon et al., 1983) using $30 \mu \mathrm{g}$ ampicillin $\mathrm{ml}^{-1}$ and $25 \mu \mathrm{g}$ kanamycin $\mathrm{ml}^{-1}$ (Sigma). A rifampicin-resistant mutant of A. hydrophila 163a was selected following overnight growth on $20 \mu \mathrm{g}$ rifampicin $\mathrm{ml}^{-1}$ (Sigma) containing nutrient agar (Oxoid, UK). This mutant was named 163aRIF. Overnight cultures of 163aRIF and S17::pUTminiKM-2 were separately grown overnight in nutrient broth (Oxoid) containing the appropriate selection and $100 \mu \mathrm{l}$ volumes of each culture were mixed together in $5 \mathrm{ml} 10 \mathrm{mM} \mathrm{MgSO}_{4}$ for $5 \mathrm{~min}$. The mixture was filtered through a $0.45 \mu \mathrm{m}$ sterile nitrocellulose filter under vacuum, and the filter was placed onto the surface of a nutrient agar plate (containing no antibiotics) and incubated at $37{ }^{\circ} \mathrm{C}$ overnight to mobilize pUT-MiniKm-2 into 163aRIF. The filter was then lifted off and shaken in $2 \mathrm{ml} 10 \mathrm{mM} \mathrm{MgSO}_{4}$ to disperse the bacteria; $200 \mu$ aliquots of the resulting suspension were plated onto the surfaces of nutrient agar plates containing $20 \mu \mathrm{g}$ rifampicin $\mathrm{ml}^{-1}$, $30 \mu \mathrm{g}$ kanamycin $\mathrm{ml}^{-1}$ and $2 \mu \mathrm{g}$ cefotaxime $\mathrm{ml}^{-1}$ (to select for $\beta$ lactamase-overproducing mutants) and the plates were incubated overnight at $37{ }^{\circ} \mathrm{C}$. On average, two colonies grew per plate. Mutants were shown to contain the Mini-Tn903- $\mathrm{Km}^{\mathrm{R}}$ cassette using PCR as described previously (Avison et al., 2000b) with primers mini-KM2 F (5'-GTAATGGCTGGCCTGTTG-3') and mini-KM2 R (5'CAACAGGCCAGCCATTAC-3').

Identification of disrupted genes. Total genomic DNA was purified and used to construct genomic libraries using a variety of single restriction enzymes (Sambrook et al., 1989) and the cloning vector pSU18 (Martinez et al., 1988). The genomic libraries were used to transform E. coli $\mathrm{DH} 5 \alpha$ (Invitrogen) to chloramphenicol resistance $\left(30 \mu \mathrm{g} \mathrm{ml}^{-1}\right)$ and recombinants containing a cloned Mini-Tn903$\mathrm{Km}^{\mathrm{R}}$ cassette were selected with kanamycin $\left(25 \mu \mathrm{g} \mathrm{ml}^{-1}\right)$. The ends of the inserts in recombinant plasmids were sequenced using M13 primers that bind either side of the multiple cloning site of pSU18 (Martinez et al., 1988). The raw sequence was used to search the $A$. hydrophila ATCC $7966^{\mathrm{T}}$ genome sequence (Seshadri et al., 2006) using BLASTN (Altschul et al., 1990) in order to identify the disrupted gene. The presence of the Mini- $\mathrm{Tn} 903-\mathrm{Km}^{\mathrm{R}}$ cassette within these genes on the chromosomes of the transposon insertion mutants was confirmed using PCR with one primer anchored in the Mini-Kn-2 cassette, KNR3 R (5'-GTATGAGTCAGCAACACC-3') or KNR 2F 


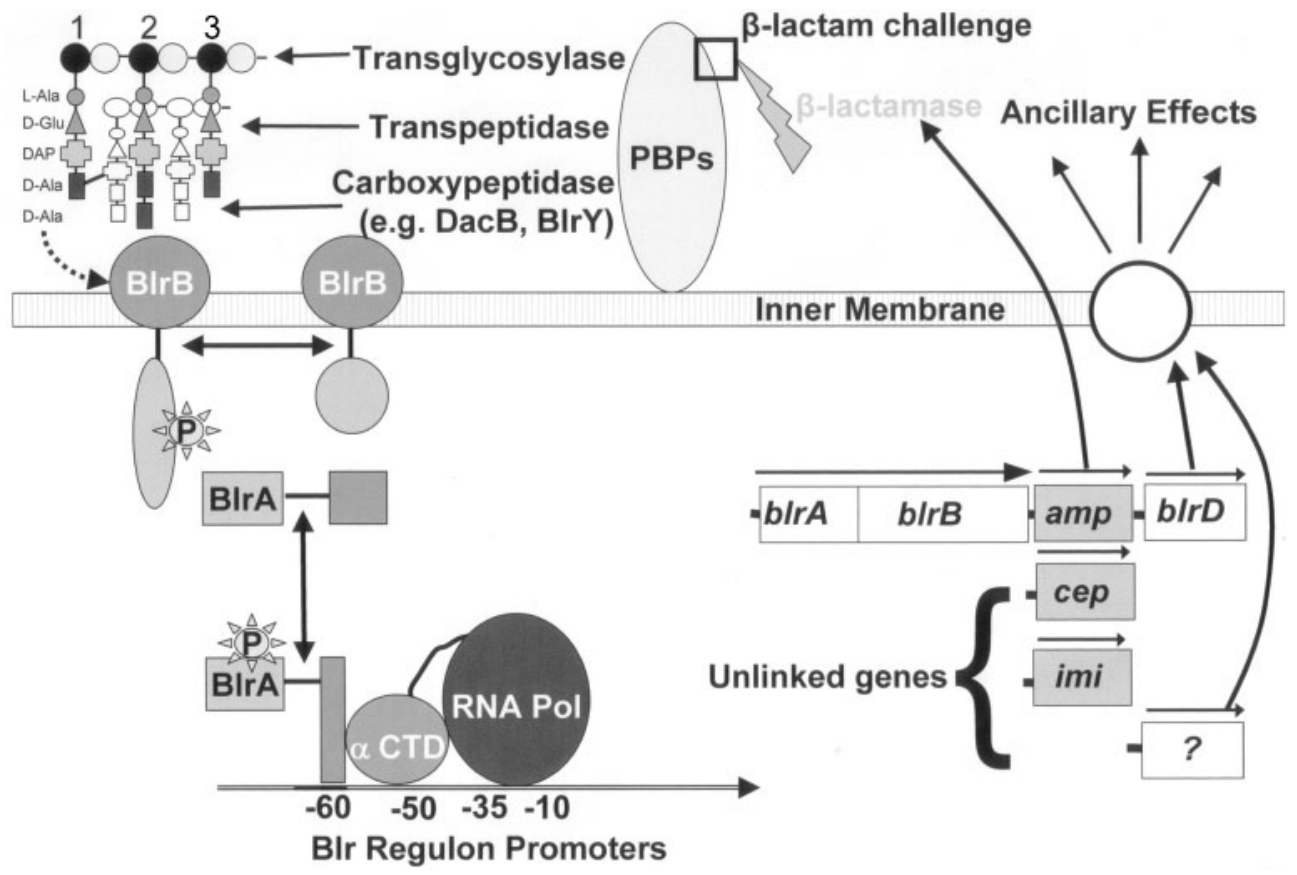

Fig. 1. Model for $\beta$-lactamase induction in Aeromonas spp. and the role of the blr regulon. PBPs are enzymes that have three major roles in peptidoglycan synthesis. High-molecular-mass, essential PBPs are transglycosylases, which join the disaccharide pentapeptides together, and/or transpeptidases, which cross-link pentapeptides from different strands of peptidoglycan through formation of a bond between the fourth amino acid, a D-alanine (D-Ala), of a donor peptide and the third, diaminopimelic acid (DAP) moiety on a recipient peptide. The result is removal of the terminal D-Ala from the donor peptide (see the crosslinked peptide labelled ' 1 ', top left). Not all pentapeptides are cross-linked (see the peptide labelled ' 2 '). Carboxypeptidases (non-essential low-molecular-mass PBPs) remove the terminal D-Ala from a peptide. The resulting tetrapeptides (see the peptide labelled ' 3 ') cannot be cross-linked by transpeptidases, so the role of these enzymes is to fine-tune the amount of cross-linking in peptidoglycan. We propose that inhibition of PBPs by $\beta$-lactams causes an increase in the concentration of the BlrB activatory ligand. This ligand then interacts with BlrB (dotted arrow), causing it to auto-phosphorylate (' $P$ ' in the diagram). This phosphate is transferred to BlrA, which binds to the cre/blr tag sequence found upstream of all blr regulon gene promoters. The effect of this is to recruit RNA polymerase and activate blr regulon transcription. Known blr regulon genes encode three $\beta$ lactamases, Amp, Cep and Imi, which directly hydrolyse the $\beta$-lactam, helping to prevent further peptidoglycan damage. The blr regulon is also known to include non- $\beta$-lactamase-encoding genes, e.g. blr $D$, and it is proposed that their products have a role in protecting the cell from $\beta$-lactam challenge through an ancillary mechanism.

(5'-GCACACCTCAGCGCTAT- $\left.3^{\prime}\right)$, and one in the disrupted gene, pbp4 F (5'-GACAACCTCTATGCCGAT-3') or blrY F (5'-GAGATGTGGTGAAGAACG-3').

Muropeptide profile analysis. Muropeptide profiles were determined after total peptidoglycan digestion, and the degree and type of cross-linking were measured as described previously (Glauner, 1988; Ursinus et al., 2004). In brief, an overnight culture of each strain grown with aeration in LB medium was diluted $1: 2000$ in $500 \mathrm{ml}$ of fresh identical medium. When cultures reached exponential phase they were centrifuged, resuspended in ice-cold $50 \mathrm{mM}$ sodium phosphate, $\mathrm{pH} 7.3$, and dropped into a boiling $6 \%(\mathrm{w} / \mathrm{v})$ SDS solution. After boiling for $15 \mathrm{~h}$, the crude sacculi were collected by ultracentrifugation, washed free of SDS with water, and digested with $\alpha$-amylase for $2 \mathrm{~h}$ at $37^{\circ} \mathrm{C}$ and with Pronase for $90 \mathrm{~min}$ at $60^{\circ} \mathrm{C}$. After the addition of $1 \%$ SDS, the sacculi were boiled for $30 \mathrm{~min}$; SDS was then removed by repeated centrifugation and resuspension. The murein samples were digested with the muramidase cellosyl. Muropeptides were analysed after reduction with sodium borohydride by separation via reversed-phase HPLC, using a C18 ODS Hypersil column and quantification of the UV absorption of the muropeptides at $204 \mathrm{~nm}$. The total amount of peptidoglycan was calculated as the sum of the absorption of all muropeptides. The muropeptide composition of the different peaks was inferred by comparison of the known chromatogram of E. coli muramidasedigested peptidoglycan at the same positions. In cases where a perfect correlation was not possible, peaks were labelled as 'unidentified'. Experiments were repeated three times and differences in the percentage values of the different peak areas were never higher than $10 \%$ of the mean value.

$\boldsymbol{\beta}$-Lactamase induction and assay. Cultures were grown in LB medium to an $\mathrm{OD}_{600}$ of 0.8 and $\beta$-lactam challenge was applied for $1 \mathrm{~h}$ with $2 \mu \mathrm{g}$ cefoxitin $\mathrm{ml}^{-1}, 20 \mu \mathrm{g}$ ampicillin $\mathrm{ml}^{-1}$ or $0.25 \mu \mathrm{g}$ imipenem $\mathrm{ml}^{-1}$, as required. Cells were then recovered by centrifugation and total $\beta$-lactamase activity was assayed as described previously (Ayala et al., 2005). For vancomycin inhibition experiments, vancomycin was added at the appropriate concentration at an $\mathrm{OD}_{600}$ of 0.3 , and cells were then grown up and challenged with $\beta$ lactam as described above. Specific activities of $\mathrm{AmpH}$, CepH and ImiH $\beta$-lactamases were determined as described previously (Avison et al., 2002) using enzyme-specific substrates. MICs of $\beta$-lactams were 
determined using agar dilution according to the standard BSAC agar dilution method (Andrews, 2001)

\section{RESULTS}

\section{Identification of genes whose disruption leads to $\beta$-lactamase hyperproduction in $A$. hydrophila}

In members of the Enterobacteriaceae with AmpR-mediated $\beta$-lactamase induction systems, locating ampD, whose loss of function leads to constitutive $\beta$-lactamase hyperproduction, ultimately led to identification of AmpR activatory ligands. This is because AmpD degrades these activatory ligands, so loss of AmpD causes activatory ligand concentration to increase (Jacobs et al., 1995; Tuomanen et al., 1991). We have previously shown that it is possible to isolate $\beta$-lactamase-hyperproducing mutants of $A$. hydrophila with wild-type blrA and blrB TCS regulator genes (Niumsup et al., 2003). So, following the AmpD/AmpR precedent, we hypothesized that these mutants carry lossof-function mutation(s) in gene(s) encoding enzymes responsible for breaking down the BlrB activatory ligand. Loss-of-function mutations in such genes would lead to a build-up of BlrB activatory ligand concentration even in the absence of $\beta$-lactam challenge, and hence a constitutive hyperproduction of $\beta$-lactamases. In order to identify genes whose disruption leads to $\beta$-lactamase hyperproduction, as a first step to identifying the BlrB activatory ligand, we used transposon insertion mutagenesis to select $\beta$-lactamasehyperproducing mutants of the well-characterized $A$. hydrophila (previously known as A. sobria - see Methods) clinical isolate 163a (Walsh et al., 1995).

Nineteen kanamycin-resistant transposon insertion mutants of A. hydrophila 163a capable of growing on $2 \mu \mathrm{g}$ ceftotaxime $\mathrm{ml}^{-1}$ were generated. Even though selection of the mutants was with cefotaxime, hydrolysed exclusively by the CepH $\beta$-lactamase (Avison et al., 2000a), all mutants overproduced all three chromosomally encoded A. hydrophila $\beta$-lactamases, $\mathrm{AmpH}, \mathrm{CepH}$ and $\mathrm{ImiH} / \mathrm{CphA}$, and were less susceptible to a range of $\beta$-lactams (Table 1 ), as has been found with all other cefotaxime-resistant mutants of Aeromonas spp. previously tested (Walsh et al., 1995, 1997).

One representative transposon-insertion mutant (named 163a-Tn-M1) was selected for further study. The mini$\mathrm{Tn} 903-\mathrm{Km}^{\mathrm{R}}$ cassette in the genome of $163 \mathrm{a}-\mathrm{Tn}-\mathrm{M} 1$, together with some surrounding A. hydrophila genomic sequence, was cloned as described in Methods. Sequencing of the cloned genomic fragment coupled with searches of the A. hydrophila complete genome sequence (Seshadri et al., 2006) revealed that the gene disrupted in 163a-Tn-M1 is $d a c B$, encoding penicillin-binding protein 4 ( $\mathrm{PBP} 4$ ), and so a presumed DD-carboxypeptidase/endopeptidase (see Fig. 1). PCR using one primer in the kanamycin cassette and one within the $d a c B$ gene confirmed that 11 out of 19 $\beta$-lactamase-hyperproducing transposon insertion mutants had this same gene disrupted, although the site of insertion was variable: between $18 \mathrm{bp} 5^{\prime}$ and $107 \mathrm{bp} 3^{\prime}$ proximal to the insert site defined in 163a-Tn-M1 (data not shown).

A second mutant (163a-Tn-M2) was selected as being representative of the eight $\beta$-lactamase-hyperproducing transposon-insertion mutants with intact $d a c B$ genes. Cloning of the mini- $\mathrm{Tn} 903-\mathrm{Km}^{\mathrm{R}}$ cassette as above revealed that in 163a-Tn-M2, a gene encoding a putative VanY-type DD-carboxypeptidase/endopeptidase (Seshadri et al., 2006) had been disrupted; we have named this gene blrY. PCR confirmed that $\operatorname{blr} Y$ is disrupted in seven out of eight $\beta$ lactamase-hyperproducing transposon-insertion mutants that have intact $d a c B$ genes; $b \operatorname{lr} Y$ is intact in the 11 mutants where $d a c B$ has been disrupted. The site of mini-Tn903- $\mathrm{Km}^{\mathrm{R}}$ cassette insertion in one of the 19 transposon-insertion mutants remains to be identified. We suspected that this mutant most likely was a randomly selected spontaneous mutant that hyperproduces $\beta$-lactamases and happens to have taken the mini-Tn903- $\mathrm{Km}^{\mathrm{R}}$ cassette onto its chromosome at an unrelated locus. Such spontaneous $\beta$-lactamase-hyperproducing mutants are readily selected from A. hydrophila 163a, as shown previously (Walsh et al., 1995), and PCR sequencing revealed that $b \operatorname{lr} B$ has a point mutation resulting in a methionine-246 to isoleucine change close to the kinase domain of BlrB, presumably constitutively activating it.

Table 1. MICs of $\beta$-lactams against $A$. hydrophila strains and their production of specific $\beta$-lactamases

\begin{tabular}{|c|c|c|c|c|}
\hline & $\begin{array}{c}\text { A. hydrophila } \\
163 \mathrm{a}\end{array}$ & $163 \mathrm{aM}$ & $\begin{array}{l}\text { 163a TnM1 } \\
\left(\text { dacB:Kan }{ }^{\mathrm{R}}\right)\end{array}$ & $\begin{array}{l}\text { 163a TnM2 } \\
\left(\text { blrY:Kan }{ }^{\mathrm{R}}\right)\end{array}$ \\
\hline \multicolumn{5}{|c|}{$\operatorname{MIC}\left(\mathrm{mg} \mathrm{l}^{-1}\right)$ of: } \\
\hline Piperacillin & $<2$ & $>256$ & 8 & 16 \\
\hline Cefotaxime & 0.25 & 8 & 4 & 4 \\
\hline Cefoxitin & 8 & 8 & 8 & 8 \\
\hline Ceftazidime & 0.25 & 8 & 1 & 2 \\
\hline Imipenem & $<2$ & 4 & 1 & 2 \\
\hline \multicolumn{5}{|c|}{ Activity (units $\mathrm{mg}^{-1}$ ) of: } \\
\hline $\mathrm{AmpH}$ & 122 & 5450 & 899 & 1024 \\
\hline CepH & $<0.1$ & 176 & 25 & 43 \\
\hline ImiH & $<0.1$ & 153 & 360 & 475 \\
\hline
\end{tabular}




\section{Muropeptide analysis of $\boldsymbol{A}$. hydrophila $\beta$ - lactamase-hyperproducing mutants and of wild- type cells treated with $\beta$-lactams}

The transposon-insertion mutagenesis screen revealed that disrupting either of two genes leads to hyperproduction of $\beta$-lactamases in A. hydrophila. The genes are both predicted to encode DD-carboxypeptidases/endopeptidases. The role of DD-carboxypeptidases is to remove the terminal Dalanine from muropeptides within peptidoglycan (Fig. 1). Therefore, it was thought possible that one effect of loss of PBP 4 or BlrY might be an increase in the concentration of pentapeptide side chains in peptidoglycan. To test if this was the case, we measured muropeptide levels in peptidoglycan taken from $A$. hydrophila 163a and from the $\operatorname{dacB}$ and $b \operatorname{lr} Y$ loss-of-function mutants: 163a-Tn-M1 and 163a-Tn-M2, respectively. As a control to show that any differences in muropeptide profile seen did not occur as a result of BlrB activation, we also measured muropeptide levels in the spontaneous $\beta$-lactamase-hyperproducing mutant $163 \mathrm{aM}$, derived from A. hydrophila 163a by a single-step selection for cefotaxime resistance (Walsh et al., 1995), which we have found using PCR sequencing carries an alanine-275 to threonine change in BlrB. This residue is in the 'H-box' portion of the protein (Niumsup et al., 2003). Hence, this is a BlrB kinase domain mutant and does not have elevated BlrB activatory ligand concentration. Its $\beta$-lactam susceptibility and $\beta$-lactamase production profiles are reported in Table 1 .

The muropeptide profiles (Table 2) revealed that the largest difference compared to the parent strain (163a) seen in the two transposon-insertion mutants, but not seen in the $163 \mathrm{aM}$ control strain, was, as expected, an increase in the proportion of monomer disaccharide-pentapeptides (M5) making up the peptidoglycan. M5 concentration almost doubled from $2.10 \%$ of total muropeptide in 163 a to $3.85 \%$ in the mutants. The only other notable increase was in disaccharide-tripeptide-disaccharide-tripeptide dimers having an L-DAP-DAP cross-link and bound to lipoprotein (D33DL), which increased 1.5-fold. Other changes did occur, but were less obvious, so the two muropeptides with the largest proportionate increases, M5 and D33DL, were investigated as possible BlrB activatory ligands first.

To do this, we next tested whether M5 or D33DL content increases occur during $\beta$-lactam challenge of $A$. hydrophila 163a proportionately with $\beta$-lactam inducing potential. We used three inducing $\beta$-lactams at half MIC concentrations (determined in liquid LB medium): ampicillin $(20 \mu \mathrm{g}$ $\left.\mathrm{ml}^{-1}\right)$, cefoxitin $\left(2 \mu \mathrm{g} \mathrm{ml}^{-1}\right)$ or imipenem $\left(0.25 \mu \mathrm{g} \mathrm{ml}^{-1}\right)$. At the concentrations used, the three compounds had different inducing potentials, with ampicillin and imipe-

Table 2. Muropeptide composition (\% of total) of the murein of $A$. hydrophila strains

M, monomers: M3, disaccharide-tripeptide; Mx1, unidentified disaccharide monomer; M4, disaccharide-tetrapeptide; Mx2, unidentified disaccharide monomer; M5, disaccharide-pentapeptide; M3L, disaccharide-tripeptide-bound to lipoprotein. D, dimers: D43D, disaccharidetetrapeptide-disaccharide-tripeptide with an L-Dap-Dap cross-link; D43, disaccharide-tetrapeptide-disaccharide-tripeptide with a D-Ala-Dap cross-link; Dx1, unidentified disaccharide dimer; D44, disaccharide-tetrapeptide-disaccharide-tetrapeptide with a D-Ala-Dap cross-link; D33DL, disaccharide-tripeptide-disaccharide-tripeptide with an L-Dap-Dap cross-link and bound to lipoprotein; D43L, disaccharide-tetrapeptidedisaccharide-tripeptide with a D-Ala-Dap cross-link and bound to lipoprotein. T, trimer: T444, disaccharide-tetrapeptide-disaccharidetetrapeptide-disaccharide-tetrapeptide with a D-Ala-Dap cross-link. N, anhidromuropeptides: D44N, disaccharide-tetrapeptide-disaccharidetetrapeptide with a D-Ala-Dap cross-link and a glycan-reducing end; T444N, disaccharide-tetrapeptide-disaccharide-tetrapeptide-disaccharidetetrapeptide with a D-Ala-Dap cross-link and a glycan-reducing end. The minimal cut-off area for the peaks to be included in the table was $0.05 \%$ of total area under the trace. Fold increases (in bold) of $\geqslant 1.5$-fold relative to 163a are shown in parentheses. Values are means of three repetitions with less than $10 \%$ variation between experiments.

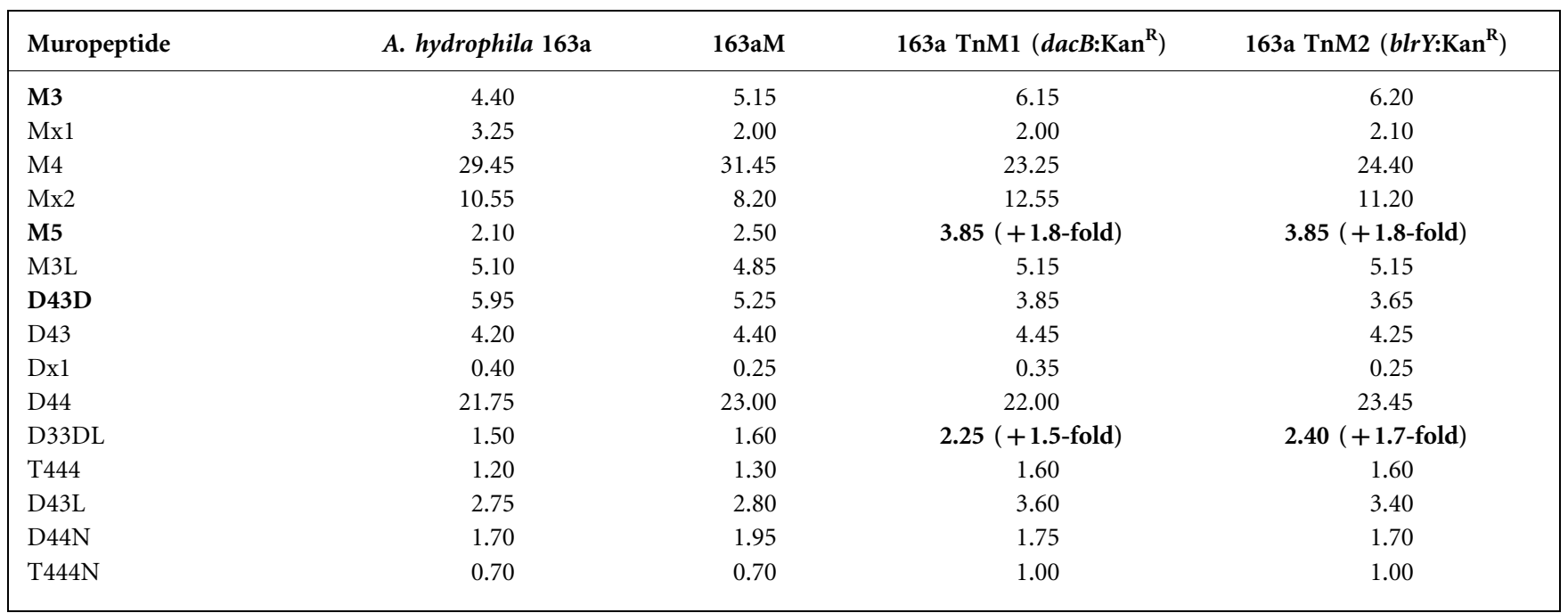


nem being very much less potent at inducing $\beta$-lactamase activity (measured using nitrocefin as a general $\beta$-lactamase substrate) than cefoxitin (Fig. 2a). Muropeptide profiling showed a large increase in both M5 and D33DL in the presence of cefoxitin, with smaller increases in the presence of ampicillin and imipenem (Fig. 2b, c), proportional with
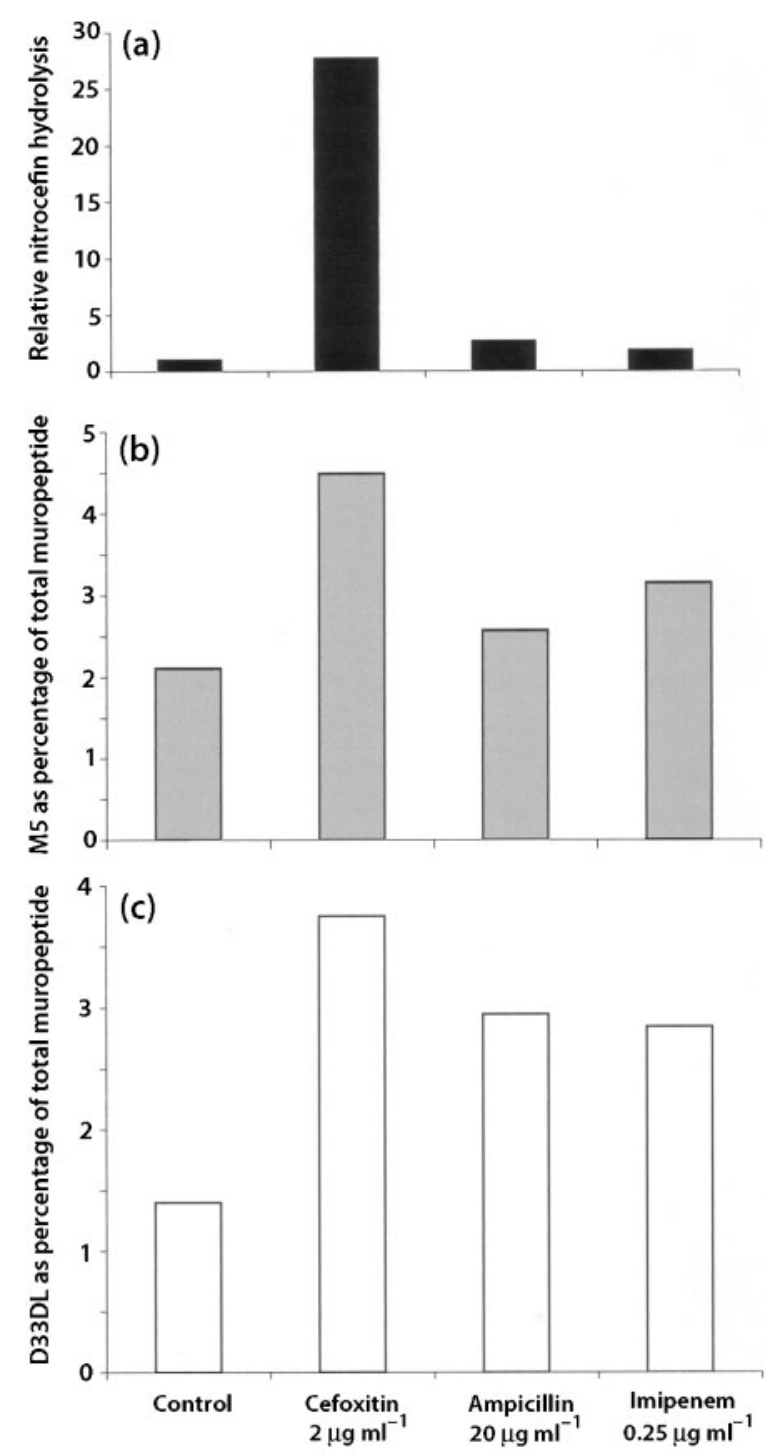

Fig. 2. Effect of $\beta$-lactam challenge on $\beta$-lactamase production and relative M5 and D33DL concentrations in A. hydrophila 163a. Cultures were grown in LB broth to mid-exponential phase and were challenged with $\beta$-lactams at the concentrations indicated (half MIC) for $1 \mathrm{~h}$. At this point, the cultures were divided. From one portion, cell extracts were prepared and total $\beta$-lactamase activity (i.e. comprising all three produced $\beta$-lactamases) was determined spectrophotometrically using nitrocefin as a substrate (a). From the other portion, muropeptides were profiled as described in the text. The percentage of M5 (b) or D33DL (c) making up peptidoglycan is shown. The data represent means of three repetitions, with less than $10 \%$ variation between repetitions. their $\beta$-lactamase induction potential. Other increases in muropeptide levels occurred, but these were not proportional with $\beta$-lactam inducing potential (Table 3 ), which is likely to reflect the slightly different binding affinities of the three $\beta$-lactams for the various functional PBP groups. Overall, therefore, these induction experiments supported our hypothesis that M5 or D33DL is the BlrB activatory ligand in A. hydrophila.

\section{Effect of vancomycin on $\beta$-lactamase induction in A. hydrophila}

Vancomycin permeates the outer membranes of Gramnegative bacteria very slowly because of its large size, meaning that cells are intrinsically resistant - in a clinical sense - to its actions. However, vancomycin can still kill Gram-negative bacteria at a clinically unobtainable concentration through the same mechanism by which it kills Gram-positive bacteria: binding to the terminal D-alanine/ D-alanine of muro-pentapeptides in peptidoglycan such as M5 (Watanakunakorn, 1984). Accordingly, to test whether M5 is the BlrB activatory ligand (and to exclude D33DL, which does not bind vancomycin), we pre-treated $A$. hydrophila 163a with vancomycin at increasing concentrations before challenging with cefoxitin at the same concentration as used for the muropeptide analysis in Fig. 2. $\beta$-Lactamase assays revealed that vancomycin exerts a concentration-dependent inhibition of $\beta$-lactamase induction in A. hydrophila 163a (Fig. 3). Addition of

Table 3. Effect of $\beta$-lactam challenge on muropeptide composition (\% of total) of the A. hydrophila 163a murein

For muropeptide abbreviations, please see the legend/footnote to Table 2. FOX, Cefoxitin; AMP, ampicillin; IMP, imipenem. Values are means of three repetitions with less than $10 \%$ variation between experiments.

\begin{tabular}{|lcccc|}
\hline Muropeptide & $\begin{array}{c}\text { A. hydrophila } \\
\text { 163a }\end{array}$ & $\begin{array}{c}\text { 163a } \\
\text { (FOX) }\end{array}$ & $\begin{array}{c}\text { 163a } \\
\text { (AMP) }\end{array}$ & $\begin{array}{r}\text { 163a } \\
\text { (IMP) }\end{array}$ \\
\hline M3 & 6.20 & 3.90 & 7.25 & 6.40 \\
Mx1 & 2.60 & 1.50 & 2.30 & 2.15 \\
M4 & 30.40 & 20.20 & 28.45 & 29.30 \\
Mx2 & 8.75 & 7.55 & 2.90 & 2.90 \\
M5 & 2.15 & 4.50 & 2.60 & 3.15 \\
M3L & 3.55 & 3.15 & 3.85 & 3.30 \\
D43D & 6.00 & 4.10 & 8.00 & 4.85 \\
D43 & 3.65 & 2.20 & 5.60 & 4.90 \\
Dx1 & 0.35 & $0.00^{*}$ & $0.00^{*}$ & 0.60 \\
D44 & 20.80 & 22.35 & 19.35 & 21.15 \\
D33DL & 1.40 & 3.75 & 2.95 & 2.85 \\
T444 & 1.40 & 1.35 & 1.20 & 1.50 \\
D43L & 1.65 & 2.00 & 2.55 & 2.45 \\
D44N & 2.45 & 1.85 & 2.40 & 2.25 \\
T444N & 0.55 & 0.95 & 0.85 & 0.80 \\
& & & & \\
\hline
\end{tabular}

${ }^{\star}$ No peak was identified on the chromatogram. 


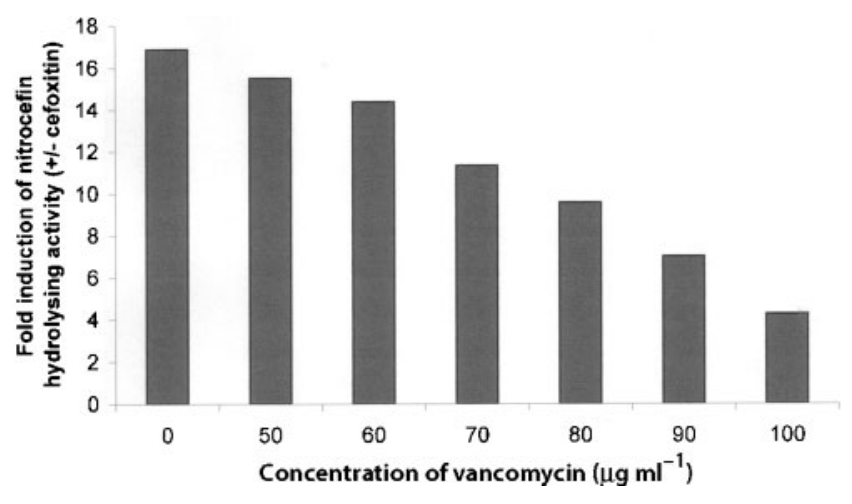

Fig. 3. Effect of vancomycin on induction of $\beta$-lactamase in the presence of cefoxitin. A. hydrophila 163a was grown overnight in LB broth in the absence of antibiotics and subcultured into LB broth to an $\mathrm{OD}_{600}$ of approximately 0.05 . The cultures were grown to an $\mathrm{OD}_{600}$ of 0.3 , at which point vancomycin was added at the concentrated indicated. One hour later, cefoxitin $\left(2 \mu \mathrm{g} \mathrm{ml}^{-1}\right)$ was added, if required. Following a further $1 \mathrm{~h}$ of growth, cell extracts were made; total $\beta$-lactamase activity in each cell extract was quantified spectrophotometrically using nitrocefin, and specific rates of hydrolysis were calculated as described previously (Avison et al., 2000a). A fold induction of $\beta$-lactamase specific activity (plus/minus cefoxitin) for each concentration of vancomycin was then calculated. The data are means of three repetitions; variation between experiments was less than $10 \%$

vancomycin to the $\beta$-lactamase-hyperproducing BlrB point mutant, $163 \mathrm{aM}$, did not reduce $\beta$-lactamase production (data not shown). These data strongly suggest that M5 and not $\mathrm{D} 33 \mathrm{DL}$, to which vancomycin will not bind, is the BlrB activatory ligand.

\section{DISCUSSION}

We have provided strong evidence that the M5 component of peptidoglycan provides the link between $\beta$-lactam challenge and $\beta$-lactamase induction in A. hydrophila. We propose that it is the activatory ligand for the periplasmic sensor domain of BlrB. Whilst we have found that an increase in the M5 content of the whole sacculus correlates with $\beta$-lactamase induction, and that induction is inhibited by vancomycin, which binds to muro-pentapeptides in peptidoglycan, we cannot totally exclude the possibility that this increase in pentapeptide could also produce an increase in periplasmic, soluble anhydro-disacharide-pentapeptide concentration. So, formally speaking, this could be a direct BlrB activatory ligand, or alternatively activation may be mediated by another M5 recognition protein, which subsequently interacts with BlrB.

In either case, this represents the third characterized mechanism that has evolved to link $\beta$-lactam challenge with $\beta$-lactamase production in bacteria. However, in only one case is a $\beta$-lactam the inducing ligand - in Bacillus spp., a relative of which is also present in Staphylococcus aureus as a horizontally acquired element (Gregory et al., 1997; Novick, 1965; Wilke et al., 2004; Zhang et al., 2001). In the well-characterized AmpR induction system (Hanson \& Sanders, 1999), and in the BlrAB induction mechanism characterized above, it is a component of peptidoglycan found at higher levels in $\beta$-lactam-challenged cells that is the activatory ligand.

Whilst there are significant differences between AmpR- and BlrAB-mediated control of $\beta$-lactamase production, in terms of both the activatory ligand and the location of induction (periplasmic for BlrAB, cytoplasmic for AmpR), there is an interesting similarity. In both systems, there is evidence that inhibition of low-molecular-mass PBPs is involved in $\beta$-lactamase induction. Disruption of such a low-molecular-mass PBP, PBP 4, strongly activates $\beta$ lactamase production in A. hydrophila, as shown above. Furthermore, it is known that $\beta$-lactams that inhibit PBP 4 are the strongest inducers of AmpR-controlled $\beta$-lactamase production in Enterobacteriaceae (Sanders et al., 1997), and in Enterobacter cloacae at least, the absolute amount of $\beta$ lactamase induction observed upon $\beta$-lactam challenge is most directly proportional to the concentration of anhydro-muramyl-pentapeptide in the cytoplasm, which will be highest if PBP 4 activity is inhibited (Dietz et al., 1997).

The observations in this study also raise the possibility that any stress causing the concentration of the BlrB activatory ligand to become elevated could switch on $\beta$-lactamase production, as has been previously suggested for other species having AmpR-mediated $\beta$-lactamase control mechanisms (Ottolenghi \& Ayala, 1991). Indeed, since in Aeromonas spp. BlrAB regulate the expression of genes in addition to those encoding $\beta$-lactamases (Avison et al., 2004), and in P. aeruginosa (at least) there is strong evidence for AmpR also being a global regulator of gene expression (Kong et al., 2005), it may be that we should start to consider these systems as envelope stress responses rather than simply $\beta$-lactam challenge responses.

Recently, it has been reported that disruption of the $d a c B$ (PBP 4) gene by point mutation in $P$. aeruginosa enhances MICs of $\beta$-lactams, and that this is the most common mechanism for elevated $\beta$-lactam MICs agains clinical $P$. aeruginosa isolates. Increased MICs occur for two reasons: first, through direct activation of AmpR-mediated AmpC $\beta$-lactamase production; and second, through activation of 'CreBC' (Moya et al., 2009). Upon annotation of the $P$. aeruginosa $\mathrm{PAO} 1$ genome sequence, this TCS operon was annotated $\mathrm{creBC}$, because of its homology with $E$. coli creBC, encoding a metabolic regulator (Avison et al., 2001), but actually, it is more similar to A. hydrophila blrAB. It appears that $P$. aeruginosa 'CreBC' is also functionally more similar to BlrAB, being activated upon PBP 4 inactivation, and having a role in $\beta$-lactam resistance (Moya et al., 2009). Interestingly, $P$. aeruginosa BlrAB does not directly control $\beta$-lactamase production. Its ancillary role in $\beta$ lactam resistance is not clear, although it is possible that 
BlrAB in Aeromonas spp. also provides this ancillary effect on $\beta$-lactam susceptibility in addition to its direct control of $\beta$-lactamase production (Avison et al., 2004).

\section{ACKNOWLEDGEMENTS}

This work was funded by grants from the Wellcome Trust (to P.M. B. and T.R.W.), the Biotechnology \& Biological Sciences Research Council (to M.B.A.), the British Society for Antimicrobial Chemotherapy (to P.M.B. and M.B.A.), the Verein zur Förderung der angewandten Mikrobiologie (to B.W.), and the European Community (LSHM-CT-2003-503335) and Spanish Ministry of Education (BFU2006-04574) (to J.A.A.). L.Z. was funded by a Society for General Microbiology Vacation Scholarship; K. W. was in receipt of a travel fellowship from the Studienstiftung des deutschen Volkes; A. E. T. was in receipt of a University of Bristol postgraduate scholarship. The authors are grateful to Dr Michael Reith, NRC Institute for Marine Biosciences, Halifax, NS, Canada, for help with preliminary analysis of Aeromonas spp. genome sequence information.

\section{REFERENCES}

Alksne, L. E. \& Rasmussen, B. A. (1997). Expression of AsbA1, OXA12 and AsbM1 $\beta$-lactamases in Aeromonas jandaei AER14 is coordinated by a two-component regulon. J Bacteriol 179, 2006-2013.

Altschul, S. F., Gish, W., Miller, W., Meyers, E. W. \& Lipman, D. J. (1990). Basic local alignment search tool. J Mol Biol 215, 403-410.

Andrews, J.M. (2001). Determination of minimum inhibitory concentrations. J Antimicrob Chemother 48 (Suppl. S1), 5-16.

Avison, M. B., Niumsup, P., Walsh, T. R. \& Bennett, P. M. (2000a). The Aeromonas hydrophila $\mathrm{AmpH}$ and $\mathrm{CepH} \beta$-lactamases: increased expression in mutants of Escherichia coli lacking creB. J Antimicrob Chemother 46, 695-702.

Avison, M. B., von Heldreich, C. J., Higgins, C. S., Bennett, P. M. \& Walsh, T. R. (2000b). A TEM $\beta$-lactamase encoded on an active Tn1like transposon in the genome of a clinical isolate of Stenotrophomonas maltophilia. J Antimicrob Chemother 46, 879-884.

Avison, M. B., Horton, R. E., Walsh, T. R. \& Bennett, P. M. (2001). Escherichia coli CreBC is a global regulator of gene expression that responds to growth in minimal media. J Biol Chem 276, 26955-26961.

Avison, M. B., Higgins, C. S., Ford, P. J., von Heldreich, C. J., Walsh, T. R. \& Bennett, P. M. (2002). Differential regulation of L1 and L2 $\beta$ lactamase expression in Stenotrophomonas maltophilia. J Antimicrob Chemother 49, 387-389.

Avison, M. B., Niumsup, P., Nurmahomed, K., Walsh, T. R. \& Bennett, P. M. (2004). Role of the 'cre/blr-tag' DNA sequence in regulation of gene expression by the Aeromonas hydrophila $\beta$-lactamase regulator, BlrA. J Antimicrob Chemother 53, 197-202.

Ayala, J., Quesada, A., Vadillo, S., Criado, J. \& Piriz, S. (2005). Penicillin-binding proteins of Bacteroides fragilis and their role in the resistance to imipenem of clinical isolates. J Med Microbiol 54, 10551064.

Cheng, Q. \& Park, J. T. (2002). Substrate specificity of the AmpG permease required for recycling of cell wall anhydro-muropeptides. J Bacteriol 184, 6434-6436.

de Lorenzo, V., Herrero, M., Jakubzik, U. \& Timmis, K. N. (1990). Mini-Tn 5 transposon derivatives for insertion mutagenesis, promoter probing, and chromosomal insertion of cloned DNA in gramnegative Eubacteria. J Bacteriol 172, 6568-6572.
Dietz, H., Pfeifle, D. \& Wiedemann, B. (1997). The signal molecule for $\beta$-lactamase induction in Enterobacter cloacae is the anhydromuramyl-pentapeptide. Antimicrob Agents Chemother 41, 2113-2120.

Glauner, B. (1988). Separation and quantification of muropeptides with high-performance liquid chromatography. Anal Biochem 172, 451-464.

Gregory, P. D., Lewis, R. A., Curnock, S. P. \& Dyke, K. G. (1997). Studies on the repressor (BlaI) of $\beta$-lactamase synthesis in Staphylococcus aureus. Mol Microbiol 24, 1025-1037.

Hanson, N. D. \& Sanders, C. C. (1999). Regulation of inducible AmpC $\beta$-lactamase expression among Enterobacteriaceae. Curr Pharm Des 5, 881-894.

Jacobs, C., Joris, B., Jamin, M., Klarsov, K., VanBeeumen, J., MenginLecreulx, D., van Heijenoort, J., Park, J. T., Normark, S. \& Frere, J. M. (1995). AmpD, essential for both $\beta$-lactamase regulation and cell wall recycling, is a novel cytosolic $\mathrm{N}$-acetylmuramyl-L-alanine amidase. Mol Microbiol 15, 553-559.

Jacobs, C., Frere, J. M. \& Normark, S. (1997). Cytosolic intermediates for cell wall biosynthesis and degradation control inducible $\beta$-lactam resistance in Gram-negative bacteria. Cell 88, 823-832.

Kong, K. F., Jayawardena, S. R., Indulkar, S. D., Del Puerto, A., Koh, C. L., Høiby, N. \& Mathee, K. (2005). Pseudomonas aeruginosa AmpR is a global transcriptional factor that regulates expression of AmpC and PoxB $\beta$-lactamases, proteases, quorum sensing, and other virulence factors. Antimicrob Agents Chemother 49, 4567-4575.

Küpfer, M., Kuhnert, P., Korczak, B. M., Peduzzi, R. \& Demarta, A. (2006). Genetic relationships of Aeromonas strains inferred from $16 \mathrm{~S}$ rRNA, gyrB and $r p o B$ gene sequences. Int J Syst Evol Microbiol 56, 2743-2751.

Lodge, J., Busby, S. \& Piddock, L. (1993). Investigation of the Pseudomonas aeruginosa ampR gene and its role in the chromosomal ampC $\beta$-lactamase promoter. FEMS Microbiol Lett 111, 315-320.

Martinez, E., Bartolome, B. \& de la Cruz, F. (1988). pACYC184derived cloning vectors containing the multiple cloning site and lacZalpha reporter gene of pUC8/9 and pUC18/19. Gene 68, 159-162.

Moya, B., Dötsch, A., Juan, C., Blázquez, J., Zamorano, L., Haussler, S. \& Oliver, A. (2009). $\beta$-Lactam resistance response triggered by inactivation of a nonessential penicillin-binding protein. PLoS Pathog 5, e1000353.

Niumsup, P., Simm, A. M., Nurmahomed, K., Walsh, T. R., Bennett, P. M. \& Avison, M. B. (2003). Genetic linkage of the penicillinase gene, $a m p$, and $b \operatorname{lr} A B$, encoding the regulator of $\beta$-lactamase expression in Aeromonas spp. J Antimicrob Chemother 51, 1351-1358.

Novick, R. P. (1965). The genetic determinant of staphylococcal penicillinase. Ann N Y Acad Sci 128, 165-182.

Okazaki, A. \& Avison, M. B. (2008). Induction of L1 and L2 $\beta$ lactamase production in Stenotrophomonas maltophilia is dependent on an AmpR-type regulator. Antimicrob Agents Chemother 52, 15251528.

Ottolenghi, A. C. \& Ayala, J. A. (1991). Induction of class I $\beta$ lactamase from Citrobacter freundii in Escherichia coli requires active $f t s Z$ but not ftsA or fts $Q$ products. Antimicrob Agents Chemother 35, 2359-2365.

Sambrook, J., Fritsch, E. F. \& Maniatis, T. (1989). Strategies for cloning in plasmid vectors. In Molecular Cloning, a Laboratory Manual, vol. 1, 2nd edn, pp. 53-104. Cold Spring Harbor, NY: Cold Spring Harbor Laboratory.

Sanders, C. C., Bradford, P. A., Ehrhardt, A. F., Bush, K., Young, K. D., Henderson, T. A. \& Sanders, W. E. (1997). Penicillin-binding proteins and induction of AmpC $\beta$-lactamase. Antimicrob Agents Chemother 41, 2013-2015. 
Sauvage, E., Kerff, F., Terrak, M., Ayala, J. A. \& Charlier, P. (2008). The penicillin-binding proteins: structure and role in peptidoglycan biosynthesis. FEMS Microbiol Rev 32, 234-258.

Seshadri, R., Joseph, S. W., Chopra, A. K., Sha, J., Shaw, J., Graf, J., Haft, D., Wu, M., Ren, Q. \& other authors (2006). Genome sequence of Aeromonas hydrophila ATCC 7966T: the Jack of all trades. J Bacteriol 188, 8272-8282.

Simon, R., Priefer, U. B. \& Puhler, A. (1983). A broad host range mobilization system for in vivo genetic engineering: transposon mutagenesis in Gram-negative bacteria. Biotechnology 1, 784-791.

Trepanier, S., Prince, A. \& Huletsky, A. (1997). Characterization of the penA and penR genes of Burkholderia cepacia 249 which encode the chromosomal class A penicillinase and its LysR-type transcriptional regulator. Antimicrob Agents Chemother 41, 2399-2405.

Tuomanen, E., Lindquist, S., Sande, S., Galleni, M., Light, K., Gage, D. \& Normark, S. (1991). Coordinate regulation of $\beta$-lactamase induction and peptidoglycan composition by the amp operon. Science 251, 201204.

Ursinus, A., van den Ent, F., Brechtel, S., de Pedro, M. A., Höltje, J.-V. \& Vollmer, W. (2004). Murein (peptidoglycan) binding property of the essential cell division protein FtsN from Escherichia coli. J Bacteriol 186, 6728-6737.

Vötsch, W. \& Templin, M. F. (2000). Characterization of a $\beta-N$ acetylglucosaminidase of Escherichia coli and elucidation of its role in muropeptide recycling and $\beta$-lactamase induction. J Biol Chem 275, 39032-39038.

Walsh, T. R., Payne, D. J., MacGowan, A. P. \& Bennett, P. M. (1995). A clinical isolate of Aeromonas sobria with three chromosomally mediated inducible $\beta$-lactamases: a cephalosporinase, a penicillinase and a third enzyme, displaying carbapenemase activity. J Antimicrob Chemother 35, 271-279.

Walsh, T. R., Stunt, R. A., Nabi, J. A., MacGowan, A. P. \& Bennett, P. M. (1997). Distribution and expression of $\beta$-lactamase genes among Aeromonas spp. J Antimicrob Chemother 40, 171-178.

Watanakunakorn, C. (1984). Mode of action and in-vitro activity of vancomycin. J Antimicrob Chemother 14 (Suppl. D), 7-18.

West, A. H. \& Stock, A. M. (2001). Histidine kinases and response regulator proteins in two-component signaling systems. Trends Biochem Sci 26, 369-376.

Wilke, M. S., Hills, T. L., Zhang, H. Z., Chambers, H. F. \& Strynadka, N. C. (2004). Crystal structures of the Apo and penicillin-acylated forms of the BlaR1 $\beta$-lactam sensor of Staphylococcus aureus. J Biol Chem 279, 47278-47287.

Zhang, H. Z., Hackbarth, C. J., Chansky, K. M. \& Chambers, H. F. (2001). A proteolytic transmembrane signalling pathway and resistance to $\beta$-lactams in Staphylococci. Science 291, 1962-1965.

Edited by: W. Bitter 